\title{
Estudio Sobre 100 Histórias Clínicas de Infertilidad
}

\author{
Dr. Alberto Arévalo Burgos*
}

\section{Consideraciones generales}

La Unidad de Fertilidad Humana de la Clínica San Pedro Claver del Instituto de Seguros Sociales, inició sus actividades en abril de 1978 como política de las Directivas del Instituto para, por un lado, orientar a la pareja afiliada con deseos de practicar planificación familiar por los diversos métodos conocidos, y, por el otro, brindar a la pareja infértil los métodos diagnósticos andrológicos y ginecológicos, tratamiento, y si no hubiere lugar a este, una orientación sobre las alternativas que pudieran quedar según la edad, la patología existente, etc.

La Unidad en su aspecto de especialidades médicas funciona de manera independiente, pero el problema de la pareja infértil es estudiado de conjunto, y al efecto, tenemos semanalmente la Reunión del Servicio, en la cual se cita una pareja que se puede ya denominar estéril por su estudio completo tanto masculino como femenino, se les explica la situación para que puedan libremente escoger las alternativas que queden; se le orienta en los aspectos sexológicos cuando por el estudio hecho lo requiera. Además, a esa reunión se llevan las historias clínicas de los dos servicios, el de ginecología y el de an-

\footnotetext{
Médico urólogo de la Unidad de Fertilidad Humana. Clinica San Pedro Claver (ISS), Bogotá
}

drología, para complementarlas mutuamente con los hallazgos encontrados. diagnósticos y tratamiento.

En la parte andrológica las consultas se han dividido así: 1.) Visita inicial en la cual se toman datos generales, se ordenan exámenes de laboratorio y dos espermogramas. 2.) Cita para examen físico y revisión de los datos de laboratorio para correlación de todos los hallazgos, hacer diagnóstico de impresión o definitivo, y programar cirugía si fue. re el caso, y 3.) Citas de control.

La historia clínica masculina que seguimos en la Unidad es (Ver Pág. 236).

\section{Material y métodos}

En este estudio de infertilidad consideramos más que todo el aspecto masculino, con correlación del femenino.

Se revisaron 242 historias clinicas, de las cuales se excluyeron 142 por incumplimiento de uno o ambos cónyuges a las consultas. Las 100 historias que quedaron comprenden un estudio desde abril $1^{\circ}$ de 1978 a septiembre $1^{\circ}$ de 1979 .

Algunos aspectos considerados en la historia clínica masculina, aunque de indudable importancia en el problema, no fueron analizados por la brevedad del tiempo asignado. 
El abandono del estudio y del tratamiento fue frecuente en este trabajo, similar al $60 \%$ encontrado por el autor en artículo anterior (1).
El modelo de espermograma seguido es el siguiente:

\section{E S P E M O G R A A}

Fecha:

Nombre

Carné No

Médico remitente

Debe enviarse a (consultorio o clínica)

Código

Edad

Hora de recolección

Días de abstinencia sexual

Caracteres generales

Aspecto

Consistencia

Licuefacción

Volumen total

Pecuento por C.C

Movilidad:
Color

Olor

$\mathrm{Ph}$ (con cifras en papel knoll)

Recuento total 24 horas

Inicial 4 horas 8 horas 24 horas

Normales

Anormales

Morfología

Formas normales \%

Anormales \% Cola $\%$ Amorfos \%, etc.

Análisis del espermograma:

Frutuosa

Test de fructolisis

Otros:

Pioesperma

Observaciones: 
Se estudiaron 93 varones afiliados al ISS, y 7 beneficiarios. De las mujeres 47 eran afiliadas y 53 beneficiarias.

\section{Edad}

Varones: Mínimo 20 a. máximo 52 a. promedio 31.98 a.

Mujeres: Mínimo 17 a. máximo 41 a. promedio 28.90 a.

\section{Escolaridad}

Varones: Primaria 79; secundaria 21.

Mujeres: Primaria 80; secundaria 20.

El tiempo de estudio comprende desde la fecha de la visita inicial hasta la fecha cuando se hizo el diagnóstico definitivo, y en caso de biopsias testiculares, hasta la fecha cuando se recibió el informe de patología. Por este detalle el tiempo podría parecer un poco largo, ya que el promedio fue de 66.3 días.

No consideramos los antecedentes familiares de mayor importancia tanto en el hombre como en la mujer.

\section{Tiempo de infertilidad previa}

Varones: Promedio 41.8 meses.

Mujeres: Promedio 41.47 meses.

De aquí en adelante se estudiarán separadamente los aspectos masculinos y femeninos.

\section{Antecedentes personales del grupo de varones}

\author{
Venéreas \\ Trauma genital \\ Orquitis por paperas \\ Orquiepididimitis venérea o \\ inespecífica \\ Orquidectomia izquierda por se- \\ minoma y radioterapia posterior \\ Vitiligo \\ Otros \\ Negativo
}

\section{Antecedentes ocupacionales}

Trabajo bajo intenso calor

Idem. y emanaciones de caucho Trabajo con fumigantes

Contacto con material radio-activo Negativo

\section{Conocimientos sobre sexología}

Nulo

Bueno

Regular

Frecuencia de relaciones sexuales

Diarias

Mensual (promedio entre 94 pacientes)

\section{Hora de las retaciones sexuales}

Entre 8 y 11 p.m.

De 5 a 6 a.m.

Variable

Posición en las relaciones sexuales

El hombre sobre la mujer

Variable

\section{Examen físico}

Normal 61

Varicocele

Atrofia testicular bilateral

Atrofia testicular unilateral

Vitiligo

Otros

\section{Exámenes de laboratorio}

\section{Normales}

Prostatitis inespecifica

Prostatitis a gonococo

Prostatitis a tricomonas

Sífilis

Diabetes

4

2

4

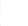

9




\section{Espermogramas}

Normales

25

Azoospermia

Oligoastenozoospermia

Oligoastenoteratozoospermia

Astenozoospermia

Astenoteratozoospermia

Teratozoospermia

Pioesperma

Dromoteratozoospermia

\section{Diagnóstico}

Fértiles

Causa inflamatoria

Varicocele

Obstructiva

Ocupacional

Orquitis urleana

Inmunológica

Sexológica
Idiopática o indeterminada

\section{Resumen de los tratamientos}

No volvieron a trat. médico o

quirúrgico

Se quedan sin hijos voluntaria-

mente

Recomendación de adopción

Inseminación artificial heteróloga

En tratamiento

\section{Biopsias testiculares}

Normales

Detención espermatogénesis

Detención espermiogénesis

Ausencia de epitelio germinal

Atrofia testicular bilateral

\section{Causales para biopsia testicular}

Astenoteratozoospermia

Oligoteratozoospermia severa

Oligoastenozoospermia

Azoospermia

Fuera de los anotados tenemos 2 pacientes con factor inflamatorio y varicocele, y uno con trauma testicular, radioterapia por tumor, hipertemia, medicamento (testosterona a altas dosis), contacto con químicos, criptorquidia, factor inflamatorio e inmunológico, trauma genital, anovulatorios suministrados al marido subrepticiamente por largo tiempo, orquitis por paperas y varicocele, y contacto con material radioactivo.

\section{Tratamiento médico}

Trat. de prostatitis

Cambio de condiciones de trabajo 7

Antialérgico y/o condón

Vitaminas $A$ y $E$, mesterolona y

hormona coriónica gonadotropa

\section{Tratamiento quirúrgico}

Varicocelectomía

Epidídimo-deferentostomía

Orquidopexia bilateral

Sexológico

Trat. de impotencia

\section{Embarazos, diagnóstico y tratamiento}

10881354 Detención espermiogénesis. Vit. A y E. (varón).

0885302 Fértil. (Mujer).

21042004 Ocupaçional. Fumigantes aéreos. Corrección factor tóxico.

Beneficiaria. Fértil.

30228400 Azoospermia. Detención germinal completa. Orquidectomía izquierda por seminoma y radioterapia posterior.

Beneficiaria. Fértil. Embarazo por inseminación artificial heteróloga.

46061119 Fértil. Corrección hábitos sexuales.

Beneficiaria. Fértil.

50388932 Oligoastenozoospermia. Varicocele. Factor inflamatorio. Detención espermiogénesis. Varicocelectomía.

1163972 Obstrucción tubárica parcial bilateral. Hidrotubaciones. 
60427758 Teratozoospermia. Factor inflamatorio. Detención espermiógenesis. Tratamiento de prostatitis

1075856 Factor cervical. Tratamientos de leucorrea.

70822773 Fértil. Corrección factor sexológico.

Benefeciaria. Fértil.

87228880 Factor inflamatorio. Varicocele. Sin tratamiento fértil.

91094023 Factor inflamatorio. Tratamiento prostatitis.

Beneficiaria. Fértil.

\section{Probables factores etiológicos}

\section{Factor cérvico vaginal}

\author{
Leucorrea \\ Cervicitis \\ Inmunológico
}

\section{Factor útero tubárico peritoneal}

Obstrucción tubárica bilateral

Obstrucción tubárica unilateral

Factor peritoneal

Endometritis y anexitis T.B.C.

\section{Factor gonadal}

Síndrome anovulatorio

Factor sexológico

Idiopática

\section{Comentarios}

De 100 estudios andrológicos se encontró patología en 75 pacientes, y en 13 parejas se encontró patología de ambos.

En nuestro concepto, el tratamiento debe ser personalizado y de acuerdo con las causales de infertilidad, masculina o femenina, halladas. Así, sería médico como en el caso de las prostatitis o factor inmunológico, y quirúrgico como en el varicocele o epidídimo-deferentostomía en azoospérmicos con trastornos oclusivos y biopsia testicular normal. Creemos que la terapia con drogas debe ser poco agresiva inicialmente, y en cuanto a las drogas de tipo hormonal, se deben prescribir individualizadas según la droga y su posología, para aumentar la dosis según respuesta. En el caso de tratamiento hormonal es deseable que el paciente tenga un estudio de plasma seminal y dosificaciones por radioinmunoanálisis de FSH, LH, y testosterona en plasma.

El trato que dé el médico a la pareja infértil es definitivo, ya que se trata generalmente de parejas angustiadas $y$ tensas por su problema. El médico debe inspirar confianza y esperanza, especialmente para que los recónditos problemas sexuales afloren y se modifiquen favorablemente con el tratamiento. Es frecuente que se cree cierta dependencia psicológica del paciente hacia el médico y que cuando la pareja aparentemente infértil decide consultar con el médico, se presente embarazo. Esto sería explicable, porque esta decisión iría acompañada de mejoría en los hábitos sexuales, especialmente en aumento de la frecuencia de las relaciones sexuales que conducen al embarazo.

No sabemos hasta dónde deba llegar el médico en su afán de mejorar las condiciones de fertilidad del paciente cuando se trata de parejas desavenidas, o en parejas en las cuales el hombre o la mujer quieren retener a la esquiva contraparte mediante un embarazo, sin modificación de las condiciones conyugales que en parte son causantes del problema de infertilidad.

Llama la atención la correlación de procesos inflamatorios a nivel de próstata y vesículas seminales en el hombre, y quizás de infección vaginal, con factor inmunológico auto o heteroinmune productor de infertilidad. Como lo demostrara Paulsen (2) hay un factor inmovilizante de los espermatozoides, obtenido del semen de individuos afectados por prostatitis por E. Coli. En nuestra opinión, otro tanto sucede con 
los individuos con prostatitis por tricomonas, en los cuales fuera del factor sanitario que representan, tienen el agravante de que para el tratamiento hay que recurrir a los derivados Nitroimidazólicos, que alteran la función del epitelio germinal testicular, aunque esta función se recupera generalmente en unos tres meses posteriores al iratamiento, y después de su curación el espermograma de control muestra una definida mejoria en el estudio comparaiivo.

En la búsqueda del factor inflamatorio como causal de infertilidad nos inclinaron los siguientes hallazgos; 1) Historia anterior de foco séptico amigdaliano u otro; 2) Historia de enfermedades venéreas tratadas o no; 3) $\mathrm{Ha}$ llazgos en el tacto rectal sugestivos de prostatitis; 4) Presencia en el espermograma de tendencia a la alcalinidad, con $\mathrm{Ph}$ de 8 ó más, aumento en el tiempo de licuefacción a más de 30 minutos, disminución del recuento espermático que puede llegar a los límites inferiores de 10 a 15 millones, pero no a la azoospermia y trastornos moderados de la movilidad espermática con límites inferiores en el $10 \%$ en el examen inicial, y presencia de pioesperma.

La biopsia testicular siempre se realizó abierta y bilateral, con exploración del contenido escrotal al mismo tiempo y se motivaron por: 1) En pacientes azoospérmicos con recuentos entre $\mathrm{O}$ y cinco millones de espermatozoides; 2) En cifras anormales de necrozoospermia; 3) En severos trastornos de la morfología espermática, bien sea por porcentajes bajos de formas normales o mul altos de una o varias anormalidades; 4) En pacientes con patología sobreagregada testicular o epididimaria que requiera tratamiento quirúrgico. Hay que tener en cuenta la presencia de una orquiepididimitis uni o bilateral traumática por la cirugía, que agravaría el problema de infertilidad del paciente. lo mismo que procesos de autoinmun.. dad desencadenados por lá misma causa.

\section{Conclusiones}

Es alarmante el porcentaje de enfermedades venéreas obtenido en los an. tecedentes personales.

Los antecedentes ocupacionales relacionados con trabajo bajo intenso calor o contacto con fumigantes $u$ otros quimicos, son de un $11 \%$ que es una cifra ya significativa.

Los conocimientos en sexología nulos en un $76 \%$, se manifiestan en frecuencia en las relaciones sexuales en exceso, o más frecuentemente en escasez con un promedio de 5.4 mensual, que ha sido causal de infertilidad en ires pacientes, pero causal coadyuvante en la mayoría. Además, las relaciones sexuales en general tienden a la monotonía, practicándose en la misma hora y en la misma posición

Indudablemente la causa inflamatoria como diagnóstico principal o coadyuvante en los problemas de infertilidad masculina entre nosotros tiene el primer puesto, y la sigue el varicocele, lo contrario de lo que sucede en ios demás paises.

La meta científica del médico dedicado al estudio y tratamiento de la infertilidad, no es solamente conseguir un embarazo en la pareja infértil, sino curar o al menos mejorar las condiciones que motivaron la infertilidad, de tal manera que la pareja pueda tener cuantos hijos quiera según su criterio personal y sin tener que recurrir a iratamiento de nuevo.

A la pareja ya comprobadamente estéril se le debe orientar y ayudar al final del estudio, sobre las alternativas que le queden, como permanecer sin hijos especailmente en parejas con unión estable' y largo tiempo de matrimonio; adopción especialmente en mujeres estériles añosas, con las implicaciones familiares y sociales que conlleva; inseminación artificial heteróloga, en el caso de hombre estéril y mujer fértil, y 
como definitiva alternativa explicándoles el pro y el contra de cada una.

En cuanto a la inseminación artificial heteróloga se deben tener en cuenta los siguientes considerandos:

1. Posibilidad de rechazo psicológico por uno de los cónyuges del hijo obtenida mediante este método o mediante adopción.

2. Ausencia de Legislación al respecto en Colombia, $y$, por ende, dificultad en la solución de los problemas que se pudieran presentar.

\section{Aspectos religiosos y morales.}

4. Los aspectos relacionados con ética profesional, fines especulativos del dador y de la entidad que practique la inseminación, y los aspectos legales. considerando que se trataría de un heteroinjerto de tejido vivo similar a una transfusión sanguinea o un injerto renal. Que yo sepa, tan sólo cursa actualmente un Proyecto de Ley en el Congreso (3) para legișlar sobre injertos. Pero es de pensar que por aspectos religiosos, dogmáticos, inmodificables, ¿vamos a cerrarle la puerta a la última oportunidad que tiene la pareja estéril y que debe ser la última en su orden? No lo creo; el hijo nacido por inseminación artificial ante la Ley sería un hijo natural si no mediara el secreto profesional, pero tendrá el hipotético $50 \%$ de las facciones de la madre y sus características corporales; la mujer se realiza como tal a través de la maternidad y del parto, el hijo podría ser registrado como hijo legítimo y podría ser bautizado, y las condiciones familiares y sociales apra él serán menos adversas que en la adopción.
Es aconsejable corroborar los resultados del espermograma con test post coito en la mujer, especialmente en presencia de espermogramas normales y condiciones ginecológicas buenas para embarazo, que lo inducen a uno a pensar en factor inmunológico, que es más frecuente de lo que se cree comolo vimos en el presente estudio, lo mismo que la autoaglutinación espermática observada en el espermograma sugestiva de trastorno inmunológico autoinmune. Cabe la posibilidad de que la presencia de leucorrea en la mujer, pueda determinar la formación de un factor inmovilizante del espermatozoide, y podría desencadenar una reacción de tipo inmunológico de rechazo del espermatozoide con aglutinación de estos, o alteración de su movilidad que de todas maneras es quizás el parámetro más importante de analizar en el espermograma.

Agradecemos la colaboración prestada por los doctores Fernando Sánchez Torres, director del Departamento de Ginecología y Obstetricia de la Clínica San Pedro Claver, Enrique Duplat Yáñez y Nelson Hugo Buriticá Ruiz, ginecólogos del Departamento, y al doctor Rafael Pinaud quien hizo el estudio de patología de la mayoría de las biopsias tomadas.

\section{Bibliografía}

1 AREVALO-BURGOS, A. Infertilidad masculina Estudio de 50 casos. Tribuna médica. Julio 1979. Pág. 21

2 PAULSEN, J. and POLAKISKY, L. (Waschington Univ.). Isolation of a Spermatozoal Inmobilization Factor from Scherichia Coli filtrates. Fertil Steril. 28: 182, 185. February 1977.

3 BLANCO-GUTIERREZ, L. A. Comunicación personal. 